\title{
Spectroscopic Determination of pKa Constants of MADS Box Segments
}

\author{
Barbora Řezáčová, ${ }^{1,2}$ Yves-Marie Coïc, ${ }^{3}$ Christian Zentz, ${ }^{2}$ \\ Pierre-Yves Turpin, ${ }^{2}$ and Josef Štěpánek ${ }^{1}$ \\ ${ }^{1}$ Faculty of Mathematics and Physics, Institute of Physics, Charles University in Prague, Ke Karlovu 5, \\ 12116 Prague 2, Czech Republic \\ ${ }^{2}$ Laboratoire Acides Nucléiques et Biophotonique, FRE 3207, Université Pierre et Marie Curie, \\ place Jussieu 4, 75252 Paris Cedex 5, France \\ ${ }^{3}$ Unité de Chimie des Biomolécules, URA 2128 CNRS, Institut Pasteur, rue du Dr. Roux 28, \\ 75724 Paris Cedex 15, France
}

Correspondence should be addressed to Barbora Řezáčová, rezacova@karlov.mff.cuni.cz

Copyright (C) 2012 Barbora Řezáčová et al. This is an open access article distributed under the Creative Commons Attribution License, which permits unrestricted use, distribution, and reproduction in any medium, provided the original work is properly cited.

\begin{abstract}
We have introduced a new promising approach for the determination of pKa constants of oligopeptide intrinsic fluorophores and spectral components referring to their differently charged states. The method is based on the factor analysis of multiwavelength spectroscopic $\mathrm{pH}$ titration data. As an illustration, we present its application on the study of short segments of the MADS box, which is a highly conserved sequence of a so-called family of transcription factors, by techniques of UV absorption and fluorescence spectroscopies. Investigated oligopeptides contain no tryptophan but one tyrosine serving as an intrinsic fluorophore and absorber. The results indicate both good sensitivity and spectroscopic selectivity of our method, which thus may be considered as a complementary technique to conventional electrochemical methods.
\end{abstract}

Keywords: Factor analysis, dissociation constant, fluorescence spectroscopy, MADS box, tyrosine

\section{Introduction}

Proteins are biological molecules that usually contain amino acids with both acidic and basic functional groups. These amino acids can be divided into several categories according to their residue charge, polarity, and hydrophobicity. Titratable amino acids of the protein may be completely exposed to solution or buried inside the 3D structure of the protein. Their pKa constants are thus influenced by protein folding and may be sensitive to protein oligomerization, binding of ligand, denaturation, and so forth.

Determination of pKa constants of proteins is usually done by electrochemical techniques (e.g., polarimetric, potentiometric, and voltammetric titration). Direct techniques such as NMR can be used on small peptides to monitor their ionization state. 
There are also several methods of computational biology, molecular modelling, and structural bioinformatics, which can be used to estimate roughly the pKa values of titratable amino acids within proteins.

In this work, we present a highly sensitive method of pKa determination (complementary to the methods mentioned previously), based on a factor analysis of spectroscopic data, obtained namely from UV-visible absorption and fluorescence measurements. Analogous methodology can though be applied to any other kind of spectroscopic data. It should be mentioned that factor analysis has already been used to determine pKa values of small organic molecules $[1,2]$; however, it has not been used to study peptides so far (to our current knowledge).

The MADS box family of transcription factors counts over 200 members playing a crucial role in the gene regulation of higher organisms. The MADS box acronym is derived from initials of four of the originally identified members of the family: MCM1, AG, DEFA, and SRF [3]. These transcription factors share a highly conserved DNA binding motif showing a wide sequence homology of 56 amino acids. Still little is known about how targeted DNAs are recognized by these transcription factors. Available structural data reveal that MADS box protein-DNA complexes have a high structure homology, but with differences in the DNA binding process [4-9].

Core SRF contains its own intrinsic fluorophores: three tyrosines with different amino-acidic environments. In order to distinguish between these tyrosines and to see the effect of diverse environments, the $\mathrm{pH}$ dependency measurements of two small segments of SRF, each containing one tyrosine, were performed by using UV absorption and fluorescence spectroscopies: ${ }_{154}$ KLLRYTTFS $_{162}$ and ${ }_{168}$ IMKKAYEL $_{175}$.

\section{Materials and Methods}

\subsection{Materials}

Synthesis of octamer oligopeptide of sequence Ac-IMKKAYEL-amid, average $M_{w}=1036.30$, was carried out according to the Fmoc/tBu solid-phase strategy [10] on a 433A peptide synthesizer (Applied Biosystems-Forster City, CA, USA), using a Fmoc Amide Resin (Applied Biosystems). Mass characterization of the purified peptide (above $98 \%$ by RP-HPLC) was consistent with the expected value.

HPLC $98 \%$ purified oligopeptide Ac-KLRRYTTFS-amid, $M_{w}=1212.43$, was purchased from Apigenex (Prague, Czech Republic). Purity and mass weight were tested by HPLC and MS.

Spectroscopically pure $\mathrm{KOH}$ and $\mathrm{HCl}$ were obtained from Normapur and MERCK.

\subsection{Optical Absorbance and Fluorescence Measurements}

Optical absorbance spectra were recorded on a Cary 3E UV-visible spectrophotometer. Fluorescence measurements were performed on a SLM Aminco-Bowman series 2 luminescence spectrometer. The excitation and emission spectral bandwidths used were $4 \mathrm{~nm}$. The optical path length was $1 \mathrm{~cm}$. Upon excitation at $276 \mathrm{~nm}$, both tyrosine and tyrosinate contribute to the fluorescence emission. All fluorescence spectra were corrected for a minor inner filter effect (optical density being bellow 0.1) [11], and contributions of Rayleigh and Raman light scattering were subtracted. Spectra were also corrected 
(slight intensity renormalization) for the small changes of concentration of oligopeptides resulting from the addition of $\mathrm{HCl}$ and/or $\mathrm{KOH}$.

Fluorescence and absorption spectra of oligopeptides were measured in the $\mathrm{pH}$ range from 3 to 11 at room temperature $\left(20^{\circ} \mathrm{C}\right)$. Concentration of the oligopeptide was $8 \cdot 10^{-5} \mathrm{M}$. Addition of $\mathrm{HCl}$ or $\mathrm{KOH}$ caused no precipitation.

Measurements of $\mathrm{pH}$ were made on a IQ Scientific Instruments $\mathrm{pH}$ meter IQ170G equipped with a stainless steel $\mathrm{pH}$ probe.

\subsection{Spectra Analysis}

To find out the individual components forming the resulting fluorescence or absorption spectra, we submitted the set of spectra $F_{i}(\lambda)$ obtained at various $\mathrm{pH}$ conditions to factor analysis, that is, singular value decomposition (SVD) algorithm decomposing a set of $N$ spectra into a set of orthogonal normalized spectral profiles $S_{j}(\lambda), j=1,2, \ldots, N$,

$$
F_{i}(\lambda)=\sum_{j=1}^{M} V_{i j} W_{j} S_{j}(\lambda)
$$

where the normalized coefficients $V_{i j}$ quote the relative portions of the $j$ th spectral profile $S_{j}(\lambda)$ in the original spectra $F_{i}(\lambda)$ [12]. The singular numbers $W_{j}$ stand for the statistical weights of spectral profiles. As the spectral components are ordered to reach a descending succession of singular values, usually a few first terms at the right side are sufficient to approximate the original spectral set within an experimental error. This value of $M$ is referred to as factor dimension. It represents the minimal number of independent components resolved in the analyzed spectral set.

In case of $M$ equal to two (which is actually the case in the present paper), the spectral components attributable to the two different components $A$ and $B$ in the spectra (from either absorption or fluorescence) can be extracted, so that

$$
F_{i}(\lambda)=F^{A}(\lambda) * C_{i}^{A}+F^{B}(\lambda) * C_{i}^{B}
$$

where $F^{A}(\lambda)$ and $F^{B}(\lambda)$ are the spectral components forming the original spectra proportionally to their current concentrations $C_{i}^{A}$ and $C_{i}^{B}$. These are given by the current $\mathrm{pH}$ value as

$$
C_{i}^{A}=\frac{1}{1+10^{\mathrm{pH}-\mathrm{pKa}}}, \quad C_{i}^{B}=C_{i}^{A} * 10^{\mathrm{pH}-\mathrm{pKa}},
$$

where $\mathrm{pKa}$ is the dissociation constant of the chromophore (here tyrosine). 
Thanks to the factor dimension of two, both $F^{A}(\lambda)$ and $F^{B}(\lambda)$ components can be well approximated as linear combinations (with coefficients $r_{j}^{A}$ and $r_{j}^{B}$ ) of the first and the second SVD spectral profiles

$$
F^{A}(\lambda)=\sum_{j=1}^{2} r_{j}^{A} * S_{j}(\lambda), \quad F^{B}(\lambda)=\sum_{j=1}^{2} r_{j}^{B} * S_{j}(\lambda)
$$

Considering the above-mentioned basic SVD formula for $F_{i}(\lambda)$ and orthogonality of SVD spectral components, we obtained a final set of $2 \times N$ equations

$$
W_{j} V_{i j}=r_{j}^{A} * \frac{1}{1+10^{\mathrm{pH}-\mathrm{pKa}}}+r_{j}^{B} *\left(1-\frac{1}{1+10^{\mathrm{pH}-\mathrm{pKa}}}\right), \quad i=1,2, \ldots N, j=1,2 .
$$

The five unknown parameters ( $\left.\mathrm{pKa}, r_{j}^{A}, r_{j}^{B} ; j=1,2\right)$ can be solved by means of a simultaneous nonlinear least-square fit of the right sides in (2.5) to the left ones.

\section{Results and Discussion}

\subsection{Absorption Spectra}

In the measured region $220-330 \mathrm{~nm}$, both oligopeptides show a characteristic peak at $275 \mathrm{~nm}$ typical for tyrosine. Noticeable changes were observed by increasing the $\mathrm{pH}$ : the absorption band moved to longer wavelength at $294 \mathrm{~nm}$, with a 1.5 -fold increase of intensity (data not shown).

\subsection{Fluorescence Spectra}

In the measured region $290-390 \mathrm{~nm}$, both oligopeptides have a characteristic peak at $303 \mathrm{~nm}$ typical for tyrosine. With an increase of the $\mathrm{pH}$, the emission significantly decreases thanks to deprotonation of tyrosine to tyrosinate (see Figure 1(a)), which has a much lower fluorescence quantum yield [13] and its emission is shifted to higher wavelengths at about $345 \mathrm{~nm}$ [14].

\subsection{Analysis of pH Dependent Fluorescence Spectra}

Results of factor analysis applied on fluorescence spectra of oligopeptide Ac-IMKKAYEL-amid are shown in Figure 1(d). We obtained a factor dimension of 2 (the same as for absorption spectra). The first spectral profile $S_{1}$ represents an average of measured spectra, and the 1 st coefficients $V_{1}$ thus describe the decrease of the overall spectral intensity as a function of $\mathrm{pH}$. The second spectral profile $S_{2}$, which is about 100 times less significant than the first one, accounts for the main spectral changes. Contribution of this profile is about zero for spectra below $\mathrm{pH} \mathrm{7,} \mathrm{while} \mathrm{above} \mathrm{this} \mathrm{pH}$ its value increases, as can be seen from the behaviour of the 2 nd coefficients $V_{2}$.

By using our method, pKa constants were determined for both fluorescence and absorption data with a good agreement for both oligopeptides. From the oligopeptide Ac-IMKKAYEL-amid absorption 


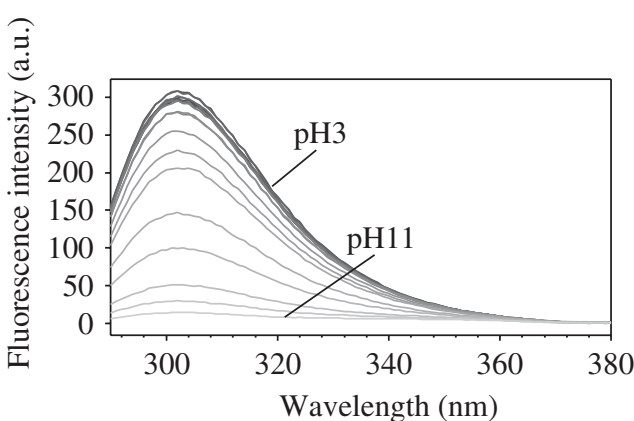

(a)

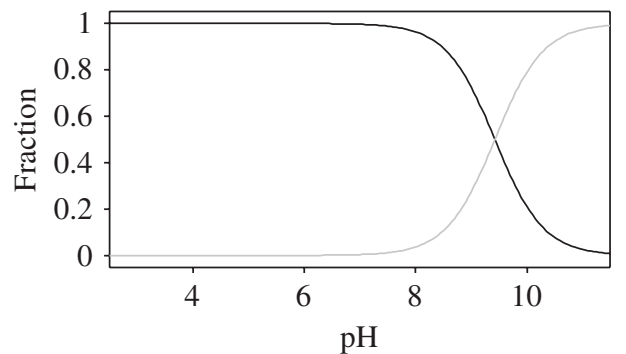

(b)

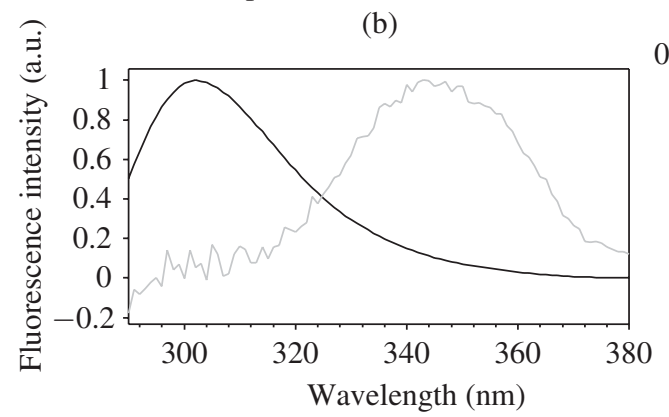

— Normalized component A - Normalized component B

(c)
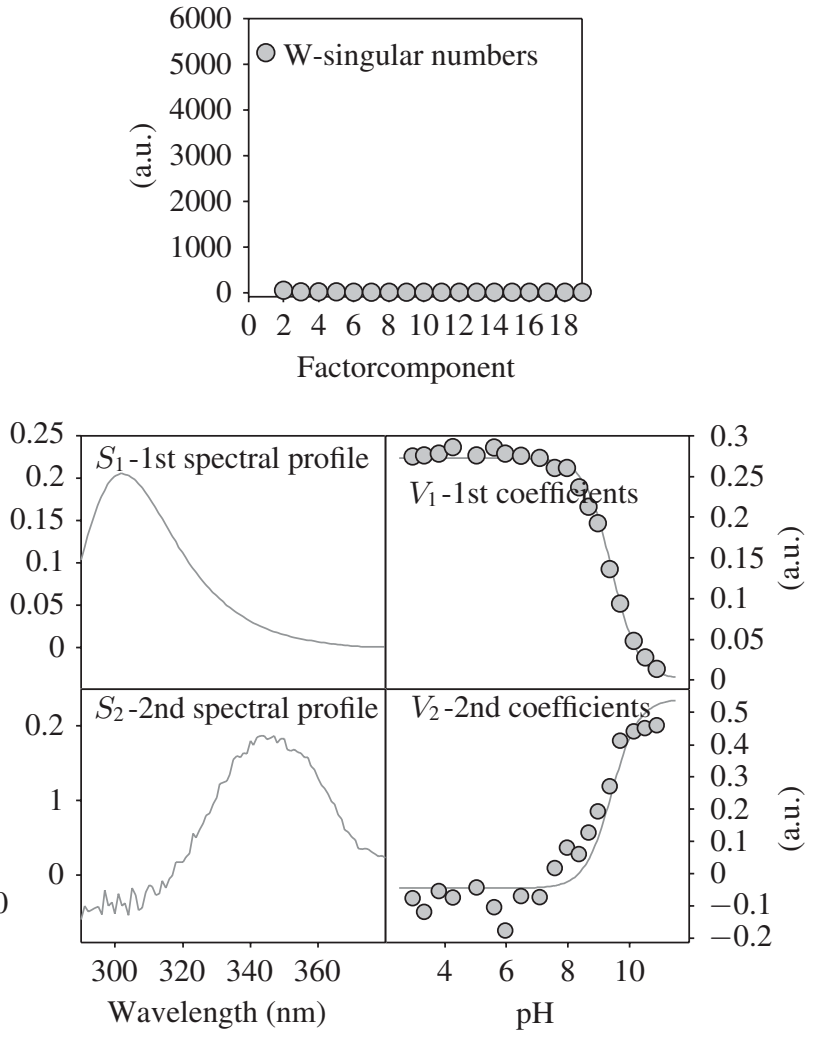

(d)

Figure 1: Fluorescence pH dependency of the MADS box segment Ac-IMKKAYEL-amid (a), determined fractions (b), normalized emission spectra of the two components-normalization factor $N_{A}=$ $0.003, N_{B}=0.201$ (c), and factor analysis results (d).

we obtained $\mathrm{pKa}=9,45 \pm 0,05$, from its fluorescence spectra $\mathrm{pKa}=9,43 \pm 0,06$. For oligopeptide Ac-KLRRYTTFS-amid absorption, we obtained $\mathrm{pKa}=9,01 \pm 0,05$, and using fluorescence spectra, we calculated $\mathrm{pKa}=9,03 \pm 0,06$. This is in good agreement with previous determinations of tyrosine pKa in various oligopeptides $[15,16]$.

In correspondence with our assumptions, we observed that the positively charged amino acids (R: arginines; K: lysines) do shift the dissociation constant of the free-state tyrosine to lower values (the dissociation constant of free tyrosine is $10.07[15,17])$. 
Moreover, using this method, we were able to distinguish the two emission components (see Figure 1(c)) and their respective fractions (Figure 1(b)). Component $A$ represents the tyrosine emission spectrum (maximum at $303 \mathrm{~nm}$ ), whereas component $B$ represents the emission of tyrosinate (maximum near $345 \mathrm{~nm})$, which is about 60 times weaker.

\section{Conclusions}

Using the factor analysis on absorption and fluorescence-monitored titration, we were able to determine the pKa constants of two tyrosine-containing oligopeptides with a high precision as well as absorption and fluorescence spectral profiles corresponding to the differently charged forms. Furthermore, we obtained quantitative information about the effect of a specific environment on $\mathrm{pKa}$ of tyrosine, which will help us to better distinguish between the different tyrosines in MADS ${ }^{\mathrm{SRF}}$.

The presented methodology is applicable to any oligopeptide bearing at least one $\mathrm{pH}$-dependent photosensitive amino acid or probe and in general can be applied to a vast number of spectroscopic techniques reflecting protonation changes in peptides. Further study on larger oligopeptide segments of SRF will be performed.

\section{Acknowledgments}

This work is supported by the Czech Science Foundation (Project 202/09/0193) and Grant Agency of Charles University (Project 402111). B. Řezáčová gratefully acknowledges the French Government support for her stay in Laboratoire Acides Nucléiques et Biophotonique.

\section{References}

[1] M. Meloun, Z. Ferenčíková, and A. Vrána, "The thermodynamic dissociation constants of methotrexate by the nonlinear regression and factor analysis of multiwavelength spectrophotometric pH-titration data," Central European Journal of Chemistry, vol. 8, no. 1, pp. 494-507, 2010.

[2] K. Y. Tam and K. Takács-Novák, "Multiwavelength spectrophotometric determination of acid dissociation constants-part II. First derivative versus target factor analysis," Pharmaceutical Research, vol. 16, pp. 374-381, 1999.

[3] P. Shore and A. D. Sharrocks, "The MADS-box family of transcription factors," European Journal of Biochemistry, vol. 229, no. 1, pp. 1-13, 1995.

[4] M. Hassler and T. J. Richmond, "The B-box dominates SAP-1-SRF interactions in the structure of the ternary complex," EMBO Journal, vol. 20, no. 12, pp. 3018-3028, 2001.

[5] K. Huang, J. M. Louis, L. Donaldson, F. L. Lim, A. D. Sharrocks, and G. M. Clore, "Solution structure of the MEF2A-DNA complex: structural basis for the modulation of DNA bending and specificity by MADS-box transcription factors," EMBO Journal, vol. 19, no. 11, pp. 2615-2628, 2000.

[6] Y. Mo, W. Ho, K. Johnston, and R. Marmorstein, "Crystal structure of a ternary SAP-1/SRF/c-fos SRE DNA complex," Journal of Molecular Biology, vol. 314, no. 3, pp. 495-506, 2001.

[7] L. Pellegrini, S. Tan, and T. J. Richmond, "Structure of serum response factor core bound to DNA," Nature, vol. 376, no. 6540, pp. 490-498, 1995. 
[8] E. Santelli and T. J. Richmond, "Crystal structure of MEF2A core bound to DNA at $1.5 \AA$ Resolution," Journal of Molecular Biology, vol. 297, no. 2, pp. 437-449, 2000.

[9] S. Tan and T. J. Richmond, "Crystal structure of the yeast MAT $\alpha 2 / M C M 1 / D N A$ ternary complex," Nature, vol. 391, no. 6668, pp. 660-666, 1998.

[10] W. C. Chan and P. D. White, Fmoc Solid Phase Peptide Synthesis: A Practical Approach, Oxford University Press, London, UK, 2000.

[11] J. R. Lakowicz, Principles of Fluorescence Spectroscopy, Springer, Singapore, 3rd edition, 2006.

[12] E. R. Malinowski, Factor Analysis in Chemistry, Wiley, New York, NY, USA, 2nd edition, 1991.

[13] J. B. A. Ross, W. R. Laws, K. W. Rousslang, and H. R. Wyssbrod, "Biochemical applications," in Topics in Fluorescence Spectroscopy, J. R. Lakowicz, Ed., vol. 3, pp. 1-63, Kluwer Academic Publishers, New York, NY, USA, 2002.

[14] A. G. Szabo, K. R. Lynn, D. T. Krajcarski, and D. M. Rayner, "Tyrosinate fluorescence maxima at $345 \mathrm{~nm}$ in proteins lacking tryptophan at pH7," FEBS Letters, vol. 94, no. 2, pp. 249-252, 1978.

[15] M. Sela and E. Katchalski, "Spectrophotometric titration of $\alpha$-amino acid copolymers containing tyrosine," Journal of the American Chemical Society, vol. 78, no. 16, pp. 3986-3989, 1956.

[16] R. L. Thurlkill, G. R. Grimsley, J. M. Scholtz, and C. N. Pace, "pK values of the ionizable groups of proteins," Protein Science, vol. 15, no. 5, pp. 1214-1218, 2006.

[17] A. L. Lehninger, D. L. Nelson, and M. M. Cox, Lehninger Principles of Biochemistry, W. H. Freeman, New York, NY, USA, 4th edition, 2004. 


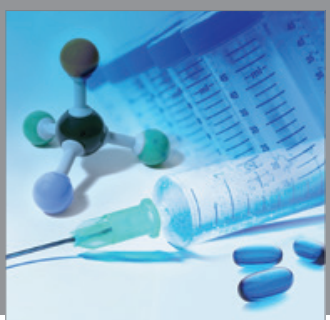

International Journal of

Medicinal Chemistry

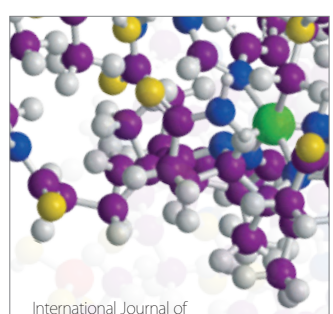

Carbohydrate Chemistry

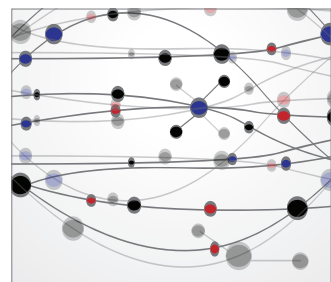

The Scientific World Journal
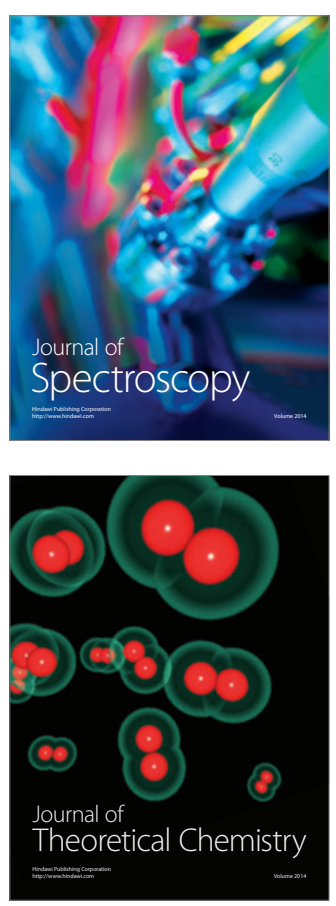
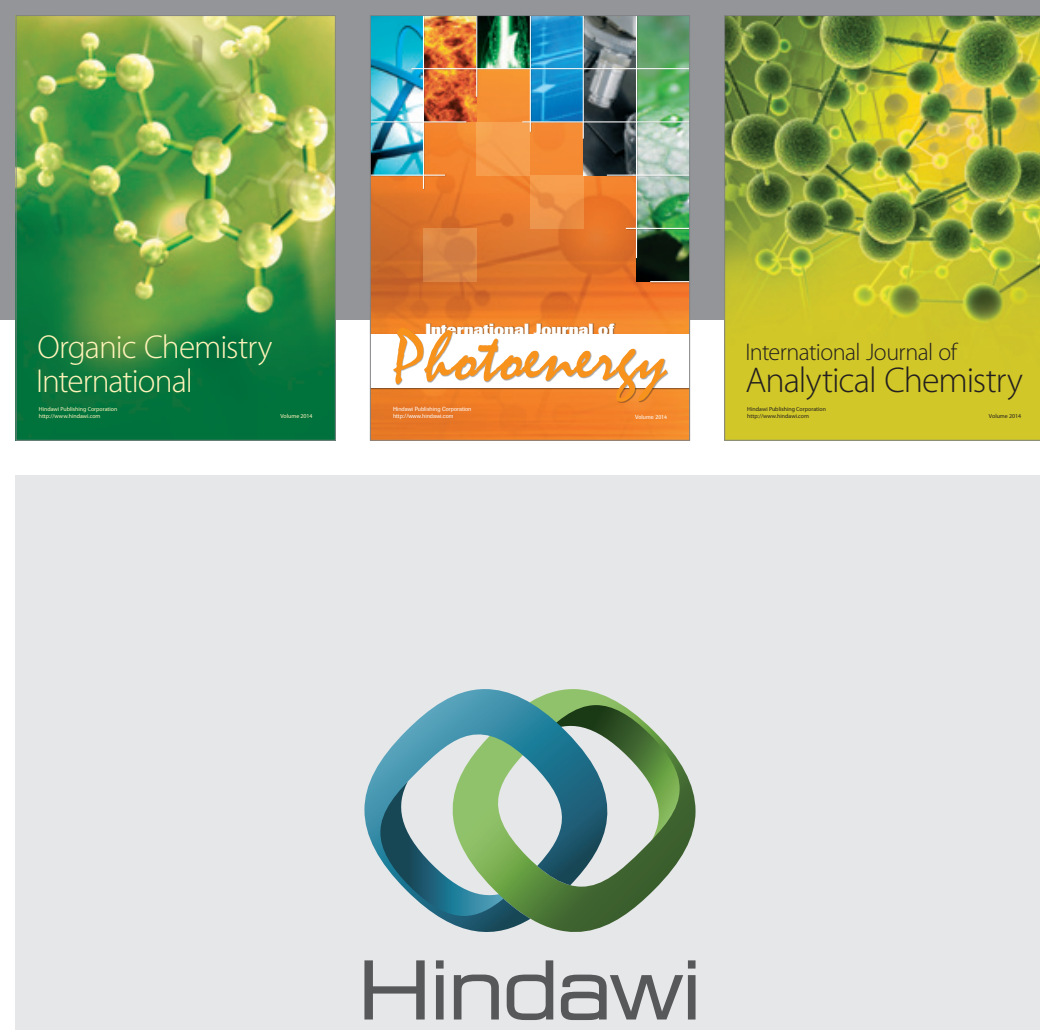

Submit your manuscripts at

http://www.hindawi.com
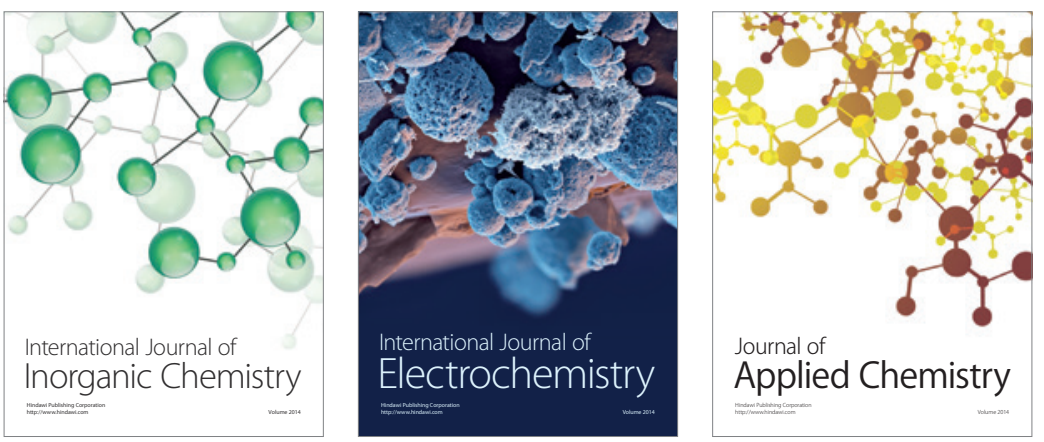

Journal of

Applied Chemistry
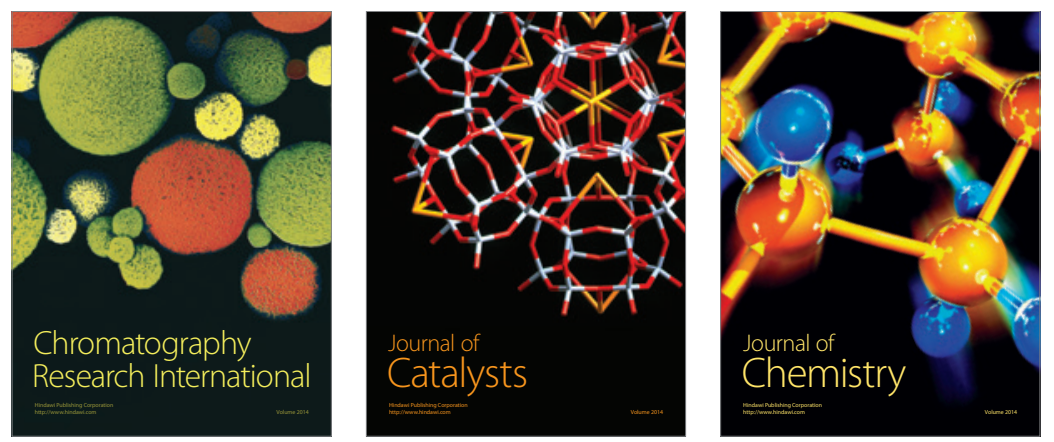
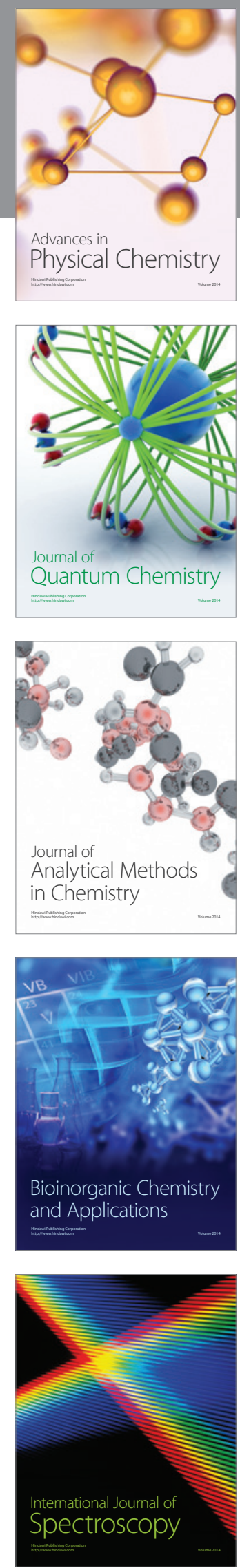